\title{
Alterations in Blood-Brain Barrier Permeability in Patients with Systemic Lupus Erythematosus
}

\author{
(D).M. Chi, (D) M. Mackay, (D)A. Hoang, (DK. Cheng, (D) C. Aranow, DJ. Ivanidze, (D) B. Volpe, DB. Diamond, and (DP.C. Sanelli
}

\begin{abstract}
BACKGROUND AND PURPOSE: Neuropsychiatric systemic lupus erythematosus refers to central and peripheral nervous system involvement, which may occur secondary to antineuronal antibodies crossing the blood-brain barrier that preferentially target cells in the hippocampus leading to abnormal hypermetabolism and atrophy. Thus, we hypothesized that alterations in BBB permeability, detected on dynamic contrast-enhanced MR imaging, occur in the hippocampus in patients with systemic lupus erythematosus before development of neuropsychiatric systemic lupus erythematosus.
\end{abstract}

MATERIALS AND METHODS: Six patients with systemic lupus erythematosus without neuropsychiatric systemic lupus erythematosus and 5 healthy controls underwent dynamic contrast-enhanced MR imaging with postprocessing into BBB permeability parameters $\left(K^{\text {trans }}\right.$ and $\mathrm{Ve}$ ) and CBF. Standardized methods selected ROI sampling of the abnormal brain regions detected on FDG-PET. The mean and SD of $K^{\text {trans }}, V e$, and CBF were calculated. Linear regression and nonparametric Spearman rank correlation analyses of $K^{\text {trans }}$ and Ve with CBF were performed. Dynamic contrast-enhanced curves and the area under the curve were generated for each brain region. Student $t$ test comparisons were performed.

RESULTS: Quantitative data revealed that patients with systemic lupus erythematosus have statistically increased $K^{\text {trans }}(P<.001)$ and Ve $(P<$ .001) compared with controls. In patients with systemic lupus erythematosus, statistically significant positive correlations were seen between $K^{\text {trans }}(P<.001)$ and $\mathrm{Ve}(P<.001)$ with CBF. Furthermore, the mean area under the curve revealed statistically increased BBB permeability in the hippocampus $(P=.02)$ compared with other brain regions in patients with systemic lupus erythematosus compared with controls.

CONCLUSIONS: These initial findings are proof-of-concept to support the hypothesis that patients with systemic lupus erythematosus have increased BBB permeability, specifically in the hippocampus, compared with other brain regions. These findings may advance our understanding of the underlying pathophysiology affecting the brain in autoimmune diseases.

ABBREVIATIONS: ANAM $=$ Automated Neuropsychological Assessment Metric; BBBP $=$ BBB permeability; DCE $=$ dynamic contrast-enhanced; DNRAb $=$ $\mathrm{N}$-methyl-D-aspartate receptor antibodies; $K^{\mathrm{trans}}=$ volume transfer constant; NMDAR $=\mathrm{N}$-methyl-D-aspartate receptor; NPSLE $=$ neuropsychiatric systemic lupus erythematosus; SLE = systemic lupus erythematosus; $V e=$ volume in the extravascular extracellular space per unit of tissue volume

S ystemic lupus erythematosus (SLE) is a chronic inflammatory autoimmune disorder characterized by a loss of immune tolerance and autoantibody production. The diverse spectrum of

Received May 7, 2018; accepted after revision December 30.

From the Department of Radiology (J.M.C., K.C.), Donald and Barbara Zucker School of Medicine at Hofstra/Northwell, Manhasset, New York; Feinstein Institute for Medical Research (M.M., C.A., B.D.), The Center for Autoimmune, Musculoskeletal and Hematopoietic Diseases, Donald and Barbara Zucker School of Medicine at Hofstra/Northwell, Manhasset, New York; Department of Radiology (A.H.), Northwell Health, Manhasset, New York; Department of Radiology (..I.), Weill Cornell Medical College, New York, New York; Feinstein Institute for Medical Research (B.V.), The Center for Biomedical Science, Donald and Barbara Zucker School of Medicine at Hofstra/Northwell, Manhasset, New York; Feinstein Institute for Medical Research (P.C.S.), The Center for Health Innovations and Outcomes Research, Donald and Barbara Zucker School of Medicine at Hofstra/ Northwell, Manhasset, New York; and Department of Radiology (P.C.S.), Northwell Health, Imaging Clinical Effectiveness and Outcomes Research Program, Manhasset, New York. symptoms reflects involvement of multiple organ systems, including musculoskeletal, renal, dermatologic, immunologic, and neurologic systems. Neuropsychiatric SLE (NPSLE) refers to central and peripheral nervous system involvement. Of the 19 clinical syndromes ${ }^{1}$ that compose NPSLE, cognitive and mood disturbances are reported most frequently, with a prevalence as high as $80 \%$ and $75 \%$, respectively, ${ }^{2}$ and, most important, have detri-

Principal Investigator: Pina Sanelli.

This work was supported by the National Institutes of Health Anti-NMDA Receptor Antibodies in Adult Brain Dysfunction and Fetal Brain Development grant, 1P01A1073693, August 2008 to July 2019

Please address correspondence to Joan Chi, MD, Donald and Barbara Zucker School of Medicine at Hofstra/Northwell, Department of Radiology, 300 Community Dr, Manhasset, NY 11030; e-mail: jchi2@northwell.edu

- Indicates open access to non-subscribers at www.ajnr.org

http://dx.doi.org/10.3174/ajnr.A5990 
mental effects on quality of life. ${ }^{3}$ Although autoantibodies are universal in patients with SLE, and circulating immune complexes have been implicated in the pathogenesis of non-NPSLE symptoms, to date, only 2 autoantibodies, anti-ribosomal $\mathrm{P}$ and cross-reactive, anti-double stranded DNA/N-methyl D-aspartate receptor antibodies (DNRAb), have been shown to directly affect neuronal function. ${ }^{4,5}$ Both anti-ribosomal $\mathrm{P}$ antibodies and DNRAb have neurotoxic effects that have been carefully studied in murine models and electrophysiologic studies; they are mediated through different mechanisms involving $N$-methyl-D-aspartate receptor (NMDAR) activation, leading to enhanced intracellular $\mathrm{Ca}++$ influx and neuronal dysfunction or death. ${ }^{6,7}$

DNRAbs, in particular, have been shown to preferentially target place cells in the CA1 region of the hippocampus and are associated with selective spatial memory deficits in the murine model. ${ }^{8}$ These results are supported by human studies in patients with SLE in which serum DNRAb titers have correlated with deficits in spatial memory testing, ${ }^{8}$ abnormal hippocampal hypermetabolism, ${ }^{9}$ and hippocampal atrophy. ${ }^{10}$ Furthermore, there have been multiple studies reported in the literature examining the CSF of patients with NPSLE, which revealed high serum antibodies within the CSF. ${ }^{11-16}$ In particular, anti-NMDAR antibodies ${ }^{13}$ and anti-NR2 subunits of NMDAR $^{14-16}$ were found in the CSF.

Despite compelling evidence for autoantibody-mediated CNS dysfunction, the caveat remains that the disruption of the BBB allows antibody access to brain tissue with subsequent neuronal alterations and cognitive loss. ${ }^{17}$ The mouse model of DNRAb-mediated cognitive dysfunction uses a nonautoimmune mouse (BALB/c strain) immunized to produce the anti-NMDAR antibodies/DNRAbs. In this model, mice with circulating DNRAbs are phenotypically intact unless lipopolysaccharide is injected intraperitoneally to mimic infection and damage the BBB. ${ }^{18}$ Following injection with lipopolysaccharide, there is evidence of DNRAb penetration in the brain with antibodies specifically binding to neurons in the hippocampus. ${ }^{18,19}$ However, little is known about BBB function in patients with SLE. On the basis of the mouse model, we hypothesized that patients with SLE may experience repeat BBB insults with time, mediated by episodes of infection, stress, hypertension, or smoking and that episodic damage to the BBB allows circulating autoantibodies, including DNRAbs, to access the brain, resulting in neuropsychiatric symptoms.

There has been increasing interest in quantitatively evaluating BBB permeability (BBBP) using dynamic contrast-enhanced (DCE) MR imaging. DCE-MR imaging is a technique in which multiple T1WI images are obtained before, during, and after contrast administration to provide signal enhancement as a function of time. ${ }^{20,21}$ DCE-MR imaging uses a 2-compartment model, specifically the intravascular space and the extravascular extracellular space, to evaluate blood flow and permeability. ${ }^{22}$ Several BBBP parameters are derived from DCE-MR imaging model-based quantitative analyses, such as volume transfer constant $\left(K^{\text {trans }}\right)$ and volume in the extravascular extracellular space per unit of tissue volume $(\mathrm{Ve}){ }^{20-22} K^{\text {trans }}$ is a flow parameter that measures the volume transfer constant from the blood plasma in the intravascular space to the extravascular extracellular space. ${ }^{23}$ A quantitative increase in the $K^{\text {trans }}$ and Ve values indicates leakage of fluid across the BBB into the brain tissue. The purpose of this study was to compare the BBBP parameters ( $K^{\text {trans }}$ and Ve), as demonstrated on DCE-MR imaging, in patients with SLE and healthy controls with specific focus on the hippocampal region.

\section{MATERIALS AND METHODS \\ Patient Cohort}

We performed a prospective study of patients with SLE and healthy controls undergoing DCE-MR imaging and clinical and neuropsychological evaluations under our current ongoing $\mathrm{Na}$ tional Institutes of Health/National Institute of Allergy and Infectious Diseases protocol (No. 1PO1A1073693). Six patients with SLE were recruited randomly from the Rheumatology Clinics at Northwell Health; all were 18 years of age or older and fulfilled the American College of Rheumatology revised criteria for SLE. ${ }^{24}$ To avoid confounding influences on neuroimaging and neuropsychological end points, key exclusion criteria included the presence of active or prior NPSLE or another CNS event; current use of antidepressant, antipsychotic, or anxiolytic drugs; or a history of excessive alcohol or illicit drug use. Additionally, subjects with SLE were required to have stable disease activity and medication doses for 4 weeks before the assessments. Disease activity and accrued damage were assessed with the Safety of Estrogens in Lupus Erythematosus National Assessment-Systemic Lupus Erythematosus Disease Activity Index (SELENA-SLEDAI; https:// www.gsksource.com/pharma/content/micro-sites/BenSELENASLEDAI/index.html ${ }^{25}$ and the Systemic Lupus International Collaborating Clinics Damage Index (SLICC DI https://slicc group.org/research/slicc-damage/) ${ }^{26}$ within 2 weeks of imaging and cognitive assessments. Cognition was assessed using the Automated Neuropsychological Assessment Metric (ANAM), ${ }^{27}$ a set of computerized measures of sustained attention, visual search, computational skills, concentration, spatial and cognitive processing, and working memory. Throughput scores, representing a combination of reaction time and accuracy, were the primary measure of cognitive efficiency used in the analyses. Subjects were additionally rated for depression and anxiety with the Beck Depression Inventory and State Trait Anxiety Inventory. ${ }^{28}$ Six ageand sex-matched healthy controls with no comorbid illness and no medications were recruited for comparison. Institutional review board approval and subject consent were obtained.

\section{Data Acquisition and Postprocessing}

All patients and controls underwent DCE-MR imaging on a 3T magnet (Siemens Prisma, Erlangen, Germany) dedicated to research at the main hospital site. MR imaging sequences included sagittal T1WI, axial T1WI, T2WI, FLAIR, and susceptibility- and diffusion-weighted imaging acquired according to standard departmental protocols. Whole-brain permeability imaging was performed using a dynamic contrast technique with axial 3D fastspoiled gradient-recalled T1WI sequences and 80 cine phases using $\mathrm{TR}=7.0 \mathrm{~ms}, \mathrm{TE}=3.08 \mathrm{~ms}, \mathrm{FOV}=24 \mathrm{~cm}$, slice thickness $=$ $5.0 \mathrm{~mm}$, and matrix size $=128 \times 256$.

The gadolinium contrast-injection protocol was standardized for all patients with SLE and healthy controls. Gadavist gadolinium contrast (gadobutrol; Bayer Schering Pharma, Berlin, Germany) was intravenously administered according to patient body weight $(0.1 \mathrm{mmol} / \mathrm{kg})$ per manufacturer's recommendations at 3 


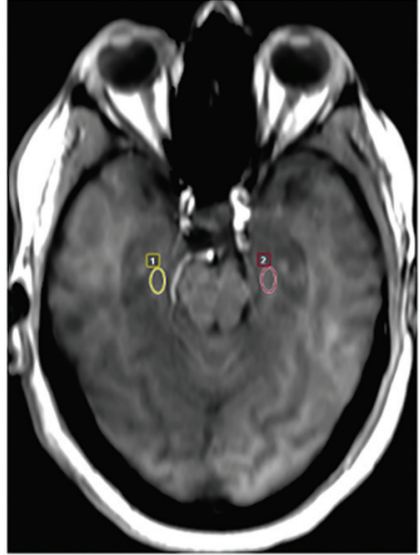

Hippocampus

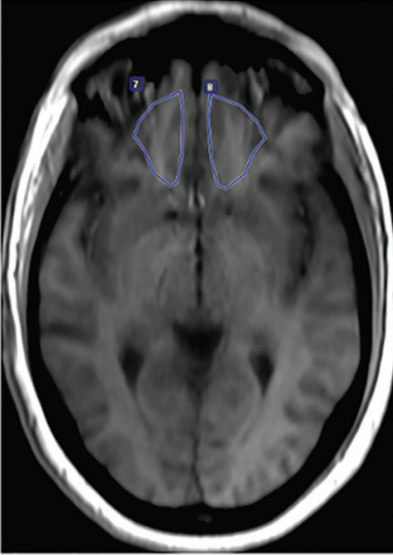

Orbitofrontal

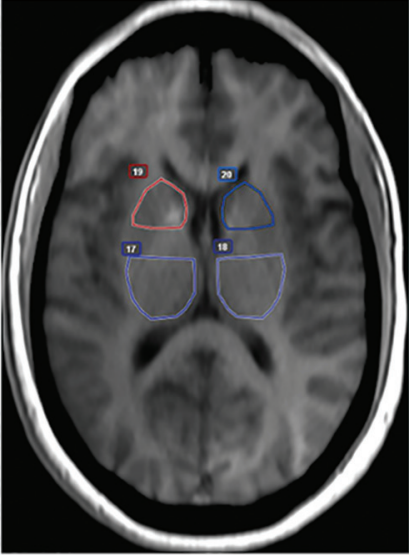

Ant Put/Caudate/Post Put GP/Thalamus

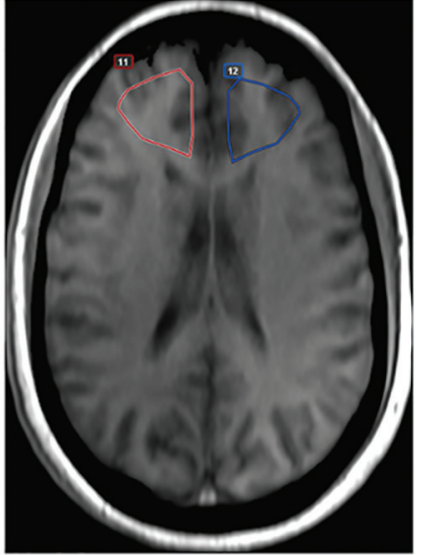

Prefrontal

FIG 1. DCE-MR imaging data acquisition methods. Mirror ROls were placed for sampling the abnormal regions described on FDG-PET: hippocampus, orbitofrontal, anterior putamen/caudate, posterior putamen/globus pallidus/thalamus, and prefrontal regions. Ant Put indicates anterior putamen; Post Put, posterior putamen; GP, globus pallidus.

$\mathrm{mL} / \mathrm{s}$ with a 45 -second delay, acquiring 5 volumes, after the DCE sequence was initiated. Immediately following contrast administration, a saline chasing bolus of 20 - $\mathrm{mL}$ volume was intravenously administered at $3 \mathrm{~mL} / \mathrm{s}$. No prebolus contrast injection was performed in this study protocol.

Postprocessing of the acquired images into BBBP parameters of $K^{\text {trans }}$ (milliliters/100 g/min), Ve (milliliters/100 g), and CBF (milliliters/100 g/min) was performed using Olea Sphere 2.2 and 2.3 (Olea Medical, La Ciotat, France) with the Tofts extended permeability model by trained research personnel. The postprocessing technique was standardized with the arterial input function placed at the center of the cavernous segment of the internal carotid artery in a similar fashion for all patients with SLE and healthy controls. A standardized method was used with selective ROI placement, sampling the following abnormal regions of metabolism described on FDG-PET ${ }^{29}$ : 1) hippocampus, 2) orbitofrontal cortex, 3) prefrontal cortex, 4) posterior putamen/globus pallidus/thalamus, and 5) anterior putamen/caudate. Mirror ROIs were placed in the cerebral hemispheres for identical sampling bilaterally at the same brain levels (Fig 1). Dynamic contrast-enhancement curves for each brain region were generated to compare the permeability phases among patients with SLE and healthy controls.

\section{Statistical Analysis}

The demographic characteristics and ANAM subtest throughput scores in patients with SLE and healthy controls were analyzed using Student $t$ tests, nonparametric Mann-Whitney $U$ tests, Pearson correlations, or Fisher exact tests as appropriate. Analyses were performed using SPSS 24 (IBM, Armonk, New York). All tests were 2 -sided, and the significance level was set at $P<.05$.

The mean and SD for each BBBP parameter ( $K^{\text {trans }}$ and Ve) and CBF were calculated for the patients with SLE and healthy controls. The overall mean $K^{\text {trans }}, \mathrm{Ve}$, and $\mathrm{CBF}$ for all regions were compared between the patients with SLE and healthy controls using the 2 -sample Student $t$ test with significance at $P<.05$.

Linear regression analyses of each BBBP factor ( $K^{\text {trans }}$ and Ve) with CBF were performed separately to evaluate the correlation coefficient $(r)$, correlation of determination $\left(R^{2}\right)$, and the statisti- cal significance in patients with SLE and healthy controls. Because a large variability among the data points of patients with SLE was observed in the scatterplots, to confirm the results of the regression analyses, we additionally analyzed the data with the nonparametric Spearman rank correlation, which is robust against nonnormally distributed data.

The mean dynamic contrast-enhancement curve for each brain region was generated in the patients with SLE and healthy controls. The area under the curve was computed for each brain region in reference to the baseline before contrast arrival. The mean area under the curve was compared in each brain region for the patients with SLE and healthy controls using the 2-sample Student $t$ test with significance at $P<.05$.

\section{RESULTS}

All 6 patients with SLE and 5 healthy controls were included in the statistical analysis. One healthy control subject was excluded due to inadequate postprocessing of the DCE-MR imaging data from excess motion. There were no significant differences between patients with SLE and healthy controls in terms of age, race, smoking, and assessments for depression and anxiety (Table 1). However, the patients with SLE demonstrated significantly lower scores on the cognitive testing than the healthy controls. Overall, the patients with SLE had low levels of disease activity, were on very little prednisone, and were all serologically negative for anticardiolipin antibodies and lupus anticoagulant, and 3 of them had hypertension controlled with medication but no other comorbid illnesses. Half of the subjects with SLE and 1 healthy control had elevated serum DNRAb titers. A comparison of mean scores for the Beck Depression Inventory, State Trait Anxiety Inventory, and ANAM tests between those with high serum DNRAb titers $(\mathrm{DNRAb}+)$ and those with low serum DNRAb titers demonstrated trends of higher depression and anxiety scores and lower throughput scores on the ANAM tests for the DNRAb+ group, though none reached statistical significance (data not shown).

The model-based quantitative data revealed that patients with SLE have statistically significant increases in $K^{\text {trans }}(P<.001)$ and 
Ve $(P<.001)$ compared with healthy controls (Table 2$)$ using all brain regions. These findings indicate that patients with SLE have increased flow across the BBB $\left(K^{\text {trans }}\right)$ coupled with accumulation of fluid in the extravascular extracellular space (Ve) in the brain.

Linear regression analyses of $K^{\text {trans }}$ and Ve with CBF, separately, demonstrated statistically significant moderate positive correlations between $K^{\text {trans }}\left(r=0.47, R^{2}=0.22, P<.001\right)$ and Ve

\begin{tabular}{|c|c|c|c|}
\hline & $\operatorname{SLE}(n=6)$ & $\begin{array}{l}\text { Healthy } \\
\text { Controls } \\
(n=5)\end{array}$ & $\begin{array}{c}P \\
\text { Value }\end{array}$ \\
\hline Age (mean) (yr) & $38.0 \pm 13.2$ & $34.2 \pm 10.5$ & .619 \\
\hline Race & & & .292 \\
\hline Caucasian & $2(33 \%)$ & 0 & \\
\hline Hispanic & 0 & $2(40 \%)$ & \\
\hline African American & $3(50 \%)$ & $2(40 \%)$ & \\
\hline Asian & $1(17 \%)$ & $1(20 \%)$ & \\
\hline Smoking & 1 & 1 & .361 \\
\hline High serum DNRAb titer & $3(50 \%)$ & $1(20 \%)$ & .545 \\
\hline \multicolumn{4}{|l|}{ ANAM testing ${ }^{a}$} \\
\hline Matching grids & $25.8 \pm 9.2$ & $40.4 \pm 10.8$ & .042 \\
\hline Match to sample & $16.4 \pm 5.9$ & $35.7 \pm 15.1$ & .043 \\
\hline Continuous processing & $65.6 \pm 5.5$ & $88.7 \pm 14.0$ & .018 \\
\hline Beck Depression Inventory & $4 \pm 4.3$ & $1.8 \pm 3.0$ & .362 \\
\hline State Trait Anxiety Inventory & $27.4 \pm 5.9$ & $25 \pm 6.9$ & .573 \\
\hline SLEDAI (mean) & $2.3 \pm 1.5$ & & \\
\hline SLICC DI (mean) & $0.5 \pm 0.8$ & & \\
\hline $\begin{array}{l}\text { Disease duration (mean) } \\
\text { (range, 1-33) (yr) }\end{array}$ & $13.3 \pm 11.1$ & & \\
\hline \multicolumn{4}{|l|}{ Medications } \\
\hline Prednisone & 1 (17\%) (5 mg QD) & & \\
\hline Immunosuppression $^{\mathrm{b}}$ & $2(33 \%)$ & & \\
\hline Hydroxychloroquine & $5(83 \%)$ & & \\
\hline
\end{tabular}

Note:-QD indicates once daily.

${ }^{a}$ Automated Neuropsychological Assessment Metric: throughput scores that represent efficiency as a function of accuracy and time.

b Both subjects were on mycophenolate mofetil; $1000 \mathrm{mg}$ QD and $500 \mathrm{mg}$ QD, respectively.

Table 2: Comparison of the mean values (SDs) for the BBBP parameters ( $K^{\text {trans }}$ and $\mathrm{Ve}$ ) using all brain regions in patients with SLE and healthy controls

\begin{tabular}{lccc}
\multicolumn{1}{c}{ BBBP Parameters } & $\begin{array}{c}\text { Patients } \\
\text { with SLE }\end{array}$ & $\begin{array}{c}\text { Healthy } \\
\text { Controls }\end{array}$ & $\begin{array}{c}\boldsymbol{P} \\
\text { Value }\end{array}$ \\
\hline$K^{\text {trans }}(\mathrm{mL} / 100 \mathrm{~g} / \mathrm{min})$ & $0.0345( \pm 0.0715)$ & $0.0008( \pm 0.0034)$ & .0004 \\
$\operatorname{Ve}(100 \mathrm{~g} / \mathrm{min})$ & $0.0920( \pm 0.1817)$ & $0.0006( \pm 0.0031)$ & .0002 \\
\hline
\end{tabular}

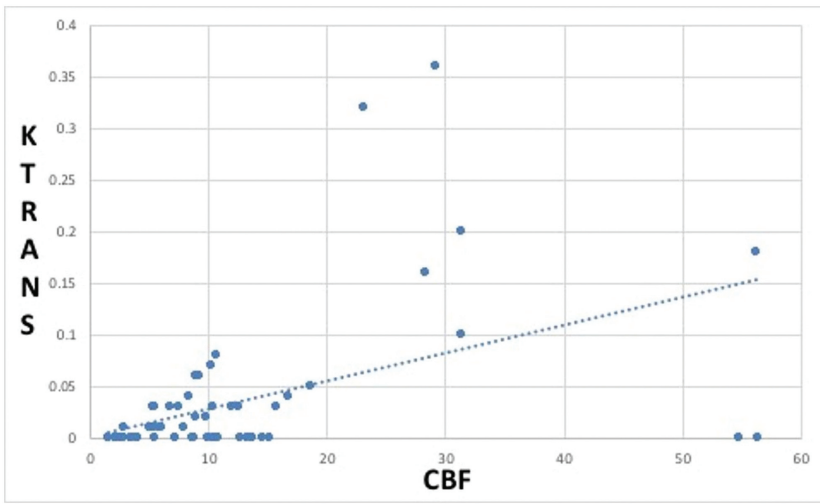

$\left(r=0.47, R^{2}=0.23, P<.001\right)$ with CBF in patients with SLE (Fig 2). In addition, the nonparametric Spearman rank correlation of $K^{\text {trans }}$ with $\mathrm{CBF}(r=0.435, P=.0005)$ and Ve with $\mathrm{CBF}(r=0.44$, $P=.0004)$ also revealed statistically significant moderate positive correlations. These findings are in contrast to the healthy controls, which demonstrate under normal conditions, as the CBF increases, $K^{\text {trans }}$ and Ve do not significantly change $(r=0.22$ and $r=0.25$ respectively) and do not correlate with $\mathrm{CBF}(P>.05)$. In addition, this data demonstrates much higher variance in the quantitative data for the patients with SLE. On the other hand, the $K^{\text {trans }}$ and Ve data in the healthy controls show much lower variance, with strong consistency in the data clustered in the same quantitative range.

When we qualitatively compared the generated mean dynamic contrast-enhancement curves in the different brain regions, patients with SLE demonstrated increased BBBP, specifically in the hippocampal region compared with healthy controls (Fig 3). Comparison of the mean area under the curve for patients with SLE and healthy controls revealed a statistically significant increased $\mathrm{BBB}$ permeability only in the hippocampal region $(P=$ $.02)$. All other brain regions demonstrated no statistically significant difference in the patients with SLE and healthy controls in the orbitofrontal $(P=.72)$, prefrontal $(P=.55)$, posterior putamen/ globus pallidus/thalamus $(P=.83)$, and anterior putamen/caudate $(P=.60)$. Furthermore, model-based quantitative data revealed that patients with SLE have statistically significant increased $K^{\text {trans }}(P=.04)$, Ve $(P=.04)$, and $\operatorname{CBF}(P=.01)$ in the hippocampal region compared with healthy controls (Table 3 ). All other brain regions did not demonstrate a statistically significant increase in both $K^{\text {trans }}$ and Ve with increased CBF $(P>.05)$.

\section{DISCUSSION}

Neuropsychiatric symptoms, mainly cognitive dysfunction and mood disturbances, frequently occur in patients with SLE, significantly impacting their quality of life. Since neurotoxic autoantibodies, DNRAb and anti-ribosomal $\mathrm{P}$ antibodies in particular, have been shown to mediate cognitive and behavioral disturbances in murine models ${ }^{6,7}$ and are associated with cognitive and behavioral dysfunctions in human SLE, ${ }^{8}$ there is increasing focus

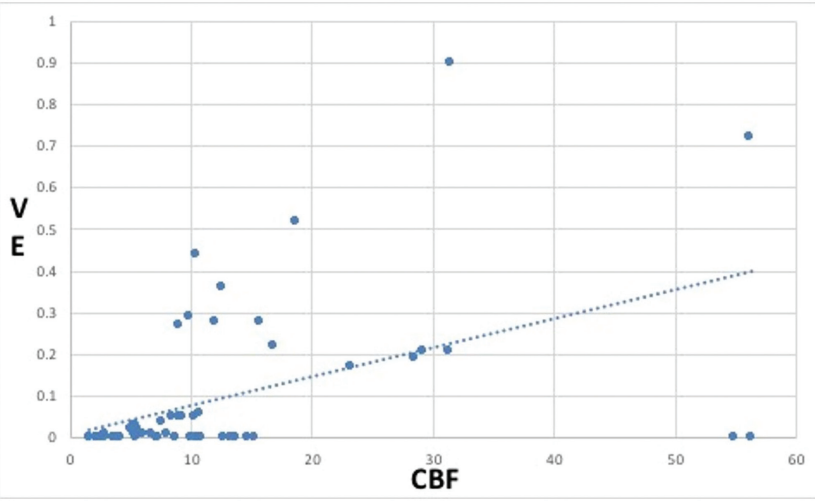

FIG 2. Linear regression analyses of the correlation of the BBBP factors ( $K^{\text {trans }}$ and Ve) with CBF using all brain regions for patients with SLE. Patients with SLE have a statistically significant moderate positive correlation between $K^{\text {trans }}$ and Ve with CBF separately. Higher variance seen in patients with SLE compared with controls may be attributed to disease- and/or treatment-related variability. Healthy controls demonstrate strong consistency in the quantitative data for both $K^{\text {trans }}$ and Ve (not shown). 

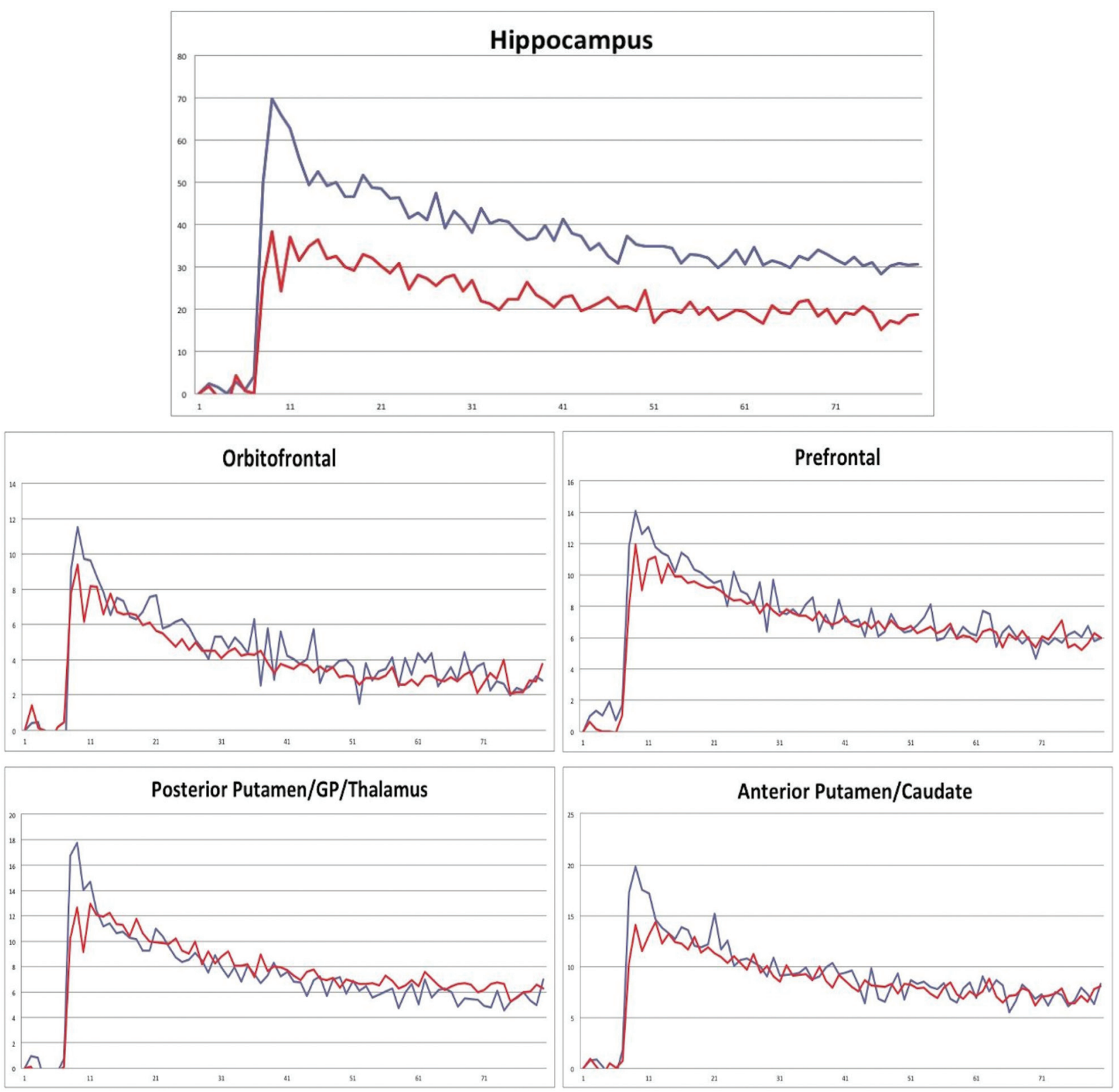

FIG 3. Dynamic contrast-enhancement curves for patients with SLE (blue) and healthy controls (red) according to the brain regions sampled. Signal intensity is represented on the $y$-axis, and the cine phase is represented on the $x$-axis. The hippocampus is the only brain region that demonstrates statistically significant increased BBB permeability $(P=.02)$ in the patients with SLE compared with the healthy controls. GP indicates globus pallidus.

Table 3: Comparison of the mean values (SD) for the BBBP parameters ( $K^{\text {trans }}$ and $\mathrm{Ve}$ ) and $\mathrm{CBF}$ in the hippocampal region in patients with SLE and healthy controls

\begin{tabular}{lccc}
\hline \multicolumn{1}{c}{ BBBP Parameters } & $\begin{array}{c}\text { Patients } \\
\text { with SLE }\end{array}$ & $\begin{array}{c}\text { Healthy } \\
\text { Controls }\end{array}$ & $\begin{array}{c}\boldsymbol{P} \\
\text { Value }\end{array}$ \\
\hline$K^{\text {trans }}(\mathrm{mL} / 100 \mathrm{~g} / \mathrm{min})$ & $0.1100( \pm 0.1331)$ & $0.019( \pm 0.0499)$ & .0405 \\
$\mathrm{Ve}(100 \mathrm{~g} / \mathrm{min})$ & $0.2000( \pm 0.3018)$ & $0.0080( \pm 0.0162)$ & .0397 \\
$\mathrm{CBF}(\mathrm{mL} / 100 \mathrm{~g} / \mathrm{min})$ & $28.84( \pm 18.82)$ & $13.14( \pm 5.89)$ & .0128 \\
\hline
\end{tabular}

on studying the BBB to understand how autoantibodies access the brain as well as to develop a potential target for treatment.

Overall, this study supports the hypothesis that patients with SLE have increased BBB permeability compared with healthy controls. The model-based quantitative data revealed that patients with SLE have statistically significant increased $K^{\text {trans }}$ and VE compared with healthy controls. These findings represent a permeable BBB profile with increased flow across the BBB (as measured by $K^{\text {trans }}$ ) coupled with the accumulation of fluid in the interstitial space in the brain (as measured by Ve). Furthermore, the linear regression and nonparametric Spearman rank analyses demonstrated that patients with SLE have statistically significant positive correlations between each BBBP parameter $\left(K^{\text {trans }}\right.$ and $\mathrm{Ve}$ ) and $\mathrm{CBF}$, indicating that leakage of fluid across the BBB is affected by $\mathrm{CBF}$ as would be expected with a permeable BBB. Thus, daily changes in $\mathrm{CBF}$, as may occur with fluctuations in systemic blood pressure, will increase flow across a permeable $\mathrm{BBB}$ in patients with SLE.

Additionally, this study supports the hypothesis that the hippocampal region specifically has increased BBB permeability in 
patients with SLE compared with healthy controls. The dynamic contrast-enhancement curves in the hippocampal region demonstrated statistically significant increased BBBP in patients with SLE. All other sampled brain regions (orbitofrontal, prefrontal, anterior putamen/caudate, and posterior putamen/globus pallidus/thalamus) did not demonstrate significant differences between patients with SLE and healthy controls. These findings were confirmed with the model-based quantitative data that revealed a statistically significant increase in both BBBP parameters in the hippocampal region in patients with SLE, representing a permeable BBB profile with increased flow across the BBB $\left(K^{\text {trans }}\right)$ coupled with accumulation of fluid in the interstitial space $(\mathrm{Ve})$ in the brain.

Several other prior studies support our findings that BBB permeability plays an important role in NPSLE. Hirohata et $\mathrm{al}^{30}$ evaluated anti-NMDAR antibody and albumin levels in CSF samples from 81 patients with SLE with active neuropsychiatric manifestations and concluded that transudation of autoantibodies through a damaged BBB plays a crucial role in NPSLE. Gulati et $\mathrm{al}^{17}$ studied 11 juvenile patients with SLE and 11 healthy controls with DCE-MR imaging and found altered BBB function in the parahippocampal gyrus, which supports our finding that the hippocampus is the area of concern in patients with SLE. ${ }^{11}$ In another study by Lauvsnes et al, ${ }^{10}$ the presence of anti-NMDAR antibodies in the CSF of patients with SLE and primary Sjögren syndrome correlated significantly with reduced hippocampal volumes on MR imaging.

This study and several others demonstrate BBB dysfunction in patients with SLE. However, there has been no established imaging technique to reliably evaluate BBB permeability. Standard CT or MR imaging can be used to infer BBB dysfunction by evaluating the presence of contrast in the brain tissue; however, evaluation of subtle leaks across the BBB is limited secondary to lack of spatial resolution. ${ }^{31} \mathrm{CSF}$ analysis can provide a quantitative assessment; however, a lumbar puncture is an invasive procedure with associated risks. FDG-PET imaging can be used to evaluate brain perfusion; however, this examination exposes patients to a high radiation dose. Dynamic-susceptibility contrast MR imaging can also be used to evaluate brain perfusion; however, many studies have reported conflicting results, demonstrating either increased CBF and CBV in patients with SLE compared with healthy controls, ${ }^{32,33}$ decreased CBV in patients with SLE, ${ }^{34}$ or no significant difference between patients with SLE and healthy controls. ${ }^{35}$ In particular, Wang et $\mathrm{al}^{32}$ evaluated 24 patients with NPSLE, 21 patients with SLE, and 21 healthy controls with DSC-MR imaging and found that patients with SLE have increased CBV and CBF in the right posterior thalamus, right hypothalamus, left parahippocampal gyrus, posterior cingulate gyrus, and left hypothalamus compared with healthy controls. In addition, Gasparovic et $\mathrm{al}^{33}$ studied DSC-MR imaging in 42 patients with SLE with 19 ageand sex-matched healthy controls and also found elevated CBF and CBV in patients with SLE in all gray and white matter of the frontal, temporal, occipital, and parietal lobes. In contrast, Zimny et $\mathrm{al}^{34}$ evaluated 22 patients with NPSLE and 13 patients with SLE and 20 age-matched healthy controls using perfusion-weighted MR imaging, which showed decreased CBV in the bilateral temporal, occipital, frontal, and parietal cortices in patients with SLE and NPSLE compared with healthy controls. Furthermore, Emmer et $\mathrm{al}^{35}$ evaluated 15 patients with active NPSLE, 26 patients with inactive NPSLE, and 11 controls with DSC-MR imaging and found that there were no differences in CBF, CBV, and MTT between patients with active or inactive NPSLE or healthy controls in the bilateral cerebral gray and white matter and thalami.

In contrast, our study used advanced imaging with dynamic contrast-enhanced MR imaging, which relies on the micron scale displacement of water molecules and can evaluate subtle changes in the capillary bed that no other imaging technique can provide with a minimal associated risk profile. ${ }^{23}$ To avoid confounding influences on BBB assessments, we also focused on subjects with SLE who had stable/low disease activity, were on low doses of corticosteroids, and had no history of acute NPSLE or other CNS events. We therefore infer that the increased BBBP identified in our study is not attributable to acute events at the time of imaging. Our study suggests that DCE-MR imaging may be an additional technique to quantitate blood-brain barrier permeability in relation to perfusion, potentially gaining further understanding of the underlying pathophysiologic mechanisms affecting the brain in patients with SLE.

Most important, this study demonstrates increased BBB permeability in patients with SLE with no history of CNS compromise but who demonstrate impaired function on tests of working memory, sustained attention, and spatial processing. Half of the subjects with SLE had elevated serum DNRAb titers as did 1 healthy control; and even in this small group, there was a trend toward lower mean test scores and higher scores for anxiety and depression as has been shown previously. ${ }^{5}$ Although patients with SLE with a known history of acute or chronic neuropsychiatric disease, particularly NPSLE, were excluded from this study, the subjects with SLE still performed worse than the healthy controls on cognitive testing. This finding highlights the prevalence of impaired cognition even in patients with SLE with no known history of neuropsychiatric disease. Increased BBB permeability that we demonstrate in this study supports the hypothesis that circulating neurotoxic autoantibodies may gain access to the CNS. The recent interest in BBB permeability as a mechanism for drug delivery to the CNS has led to an appreciation of the numerous instances in which the BBB may be compromised as a result of normal aging, microvascular disease, infection, stress, uncontrolled hypertension, toxic exposures, and neuroinflammatory states in which inflammatory molecules produced by activated microglial cells damage the $\mathrm{BBB}^{36,37}$

While we have controlled for many of these conditions in our small study, we were not able to control for medication effects on the BBB. At the time of imaging, only 2 patients with SLE were on immunosuppressive medications (mycophenolate mofetil at doses of 1000 and $500 \mathrm{mg}$ ) and only 1 patient was on prednisone, $5 \mathrm{mg}$ daily. Although high doses of corticosteroids have been associated with psychosis and altered cognition, there is much controversy over possible side effects of moderate corticosteroid use. A low dose of $5 \mathrm{mg}$ daily of prednisone should not have significant cognition-altering side effects. Two of the 6 patients were on disease-modifying drugs at low doses of 1000 and $500 \mathrm{mg}$ daily of mycophenolate mofetil, which is not known to have any cognition-altering effects. Five of 6 patients with SLE were on hydroxy- 
chloroquine, which is not immunosuppressive and also is not associated with cognition-altering effects. However, future DCE-MR imaging studies on newly diagnosed patients with SLE would provide critical information regarding medication effects on the BBB.

This initial study has several limitations, most important, the small sample size. Thus, we cannot completely exclude the possibility of false-positives on the primary analyses performed between patients with SLE and healthy controls. Nonetheless, the proof-of-concept findings of this study provide the first evidence supporting the hypothesis that patients with SLE have increased $\mathrm{BBBP}$ in the hippocampus relative to other brain regions. The quantitative data from patients with SLE demonstrated higher variances compared with healthy controls, which may be due to disease-related variability such as differences in disease severity or duration, treatment regimen, and response to treatment. Given our small sample size, further subanalyses could not be performed. A larger prospective study is necessary to confirm our findings and further investigate other subanalyses.

\section{CONCLUSIONS}

DCE-MR imaging quantitative BBBP parameters may be used as a noninvasive method to indicate a permeable BBB profile, demonstrating increased flow across the BBB (as measured by $K^{\text {trans }}$ ) coupled with accumulation of fluid in the interstitial tissue (as measured by Ve) in the brain. These initial data are proof-ofconcept to support our hypothesis that the BBB is selectively compromised, particularly in the hippocampal region, in subjects with SLE with little to no disease activity and no history of CNS insult who demonstrate impaired performance on cognitive testing. The significance of these findings may advance our understanding of the underlying pathophysiologic mechanisms affecting the brain in autoimmune diseases. Most important, larger studies are necessary to validate these results and confirm the value of DCE-MR imaging methodology as a potential biomarker for BBB permeability imaging.

Disclosures: Joan M. Chi—RELATED: Grant: National Institutes of Health/National Institute of Allergy and Infectious Diseases protocol No. 1PO1A1073693.* Meggan Mackay—RELATED: Grant: National Institute of Allergy and Infectious Diseases, National Institutes of Health, Comments: research grant 1PO1A1073693, August 2008 to July 2019*; Support For Travel to Meetings for the Study or Other Purposes: National Institute of Allergy and Infectious Diseases, National Institutes of Health, Comments: research grant IPO1A1073693, August 2008 to July 2019*; UNRELATED: Grants/Grants Pending: National Institute of Allergy and Infectious Diseases, National Institutes of Health, Comments: research grant 1PO1A1073693, August 2008 to July 2019 and research grant 1UM1A1110494, May 2014 to April 2019.* Angela HoangRELATED: Grant: National Institutes of Health. ${ }^{*}$ Katherine Cheng—RELATED: Grant: National Institutes of Health/National Institute of Allergy and Infectious Diseases protocol No. IPOIA1073693.* Jana Ivanidze_UNRELATED: Employment: Weill Cornell Medicine, Comments: I am employed as assistant professor in radiology; Grants/Grants Pending: American Society of Neuroradiology, Comments: Research Scholar Award. Bruce Volpe-RELATED: Grant: Feinstein Institute for Medical Research, Comments: National Institutes of Health grant noted in the funding section.* Betty Diamond-RELATED: Grant: National Institutes of Health.* *Money paid to the institution.

\section{REFERENCES}

1. Gulinello M, Wen J, Putterman C. Neuropsychiatric symptoms in lupus. Psychiatr Ann 2012;42:322-28 CrossRef Medline

2. Rayes HA, Tani C, Kwan A, et al. What is the prevalence of cognitive impairment in lupus and which instruments are used to measure it?
A systematic review and meta-analysis. Semin Arthritis Rheum 2018; 48:240-55 CrossRef Medline

3. Calderón J, Flores P, Aguirre JM, et al. Impact of cognitive impairment, depression, disease activity, and disease damage on quality of life in women with systemic lupus erythematosus. Scand J Rheumatol 2017;46:273-80 CrossRef Medline

4. González A, Massardo L. Antibodies and the brain: antiribosomal P protein antibody and the clinical effects in patients with systemic lupus erythematosus. Curr Opin Neurol 2018;31:300-05 10.1097/ WCO.0000000000000549 Medline

5. Mader S, Brimberg L, Diamond B. The role of brain-reactive autoantibodies in brain pathology and cognitive impairment. Front Immunol 2017;8:1101 CrossRef Medline

6. Bravo-Zehnder M, Toledo EM, Segovia-Miranda F, et al. Anti-ribosomal $P$ protein autoantibodies from patients with neuropsychiatric lupus impair memory in mice. Arthritis Rheumatol 2015;67: 204-14 CrossRef Medline

7. Segovia-Miranda F, Serrano F, Dyrda A, et al. Pathogenicity of lupus anti-ribosomal $P$ antibodies: role of cross-reacting neuronal surface $P$ antigen in glutamatergic transmission and plasticity in a mouse model. Arthritis Rheumatol 2015;67:1598-610 CrossRef Medline

8. Chang EH, Volpe BT, Mackay M, et al. Selective impairment of spatial cognition caused by autoantibodies to the N-methyl-D-aspartate receptor. EBioMedicine 2015;2:755-64 CrossRef Medline

9. Mackay M. Lupus brain fog: a biologic perspective on cognitive impairment, depression, and fatigue in systemic lupus erythematosus. Immunol Res 2015;63:26-37 CrossRef Medline

10. Lauvsnes MB, Beyer MK, Kvaløy JT, et al. Association of hippocampal atrophy with cerebrospinal fluid antibodies against the NR2 subtype of the N-methyl-D-aspartate receptor in patients with systemic lupus erythematosus and patients with primary Sjögren's syndrome. Arthritis Rheumatol 2014;66:3387-94 CrossRef Medline

11. Diamond B, Volpe BT. Antibodies and brain disease: a convergence of immunology and physiology. PLoS Med 2006;3:e498 CrossRef Medline

12. Sharp CD, Hines I, Houghton J, et al. Glutamate causes a loss in human cerebral endothelial barrier integrity through activation of NMDA receptor. Am J Physiol Heart Circ Physiol 2003;285:H2592-98 CrossRef Medline

13. Fragoso-Loyo H, Cabiedes J, Orozco-Narváez A, et al. Serum and cerebrospinal fluid autoantibodies in patients with neuropsychiatric lupus erythematosus: implications for diagnosis and pathogenesis. PLoS One 2008;3:e3347 CrossRef Medline

14. Arinuma Y, Yanagida T, Hirohata S. Association of cerebrospinal fluid anti-NR2 glutamate receptor antibodies with diffuse neuropsychiatric systemic lupus erythematosus. Arthritis Rheum 2008;58: 1130-35 CrossRef Medline

15. Gono T, Kawaguchi Y, Kaneko H, et al. Anti-NR2A antibody as a predictor for neuropsychiatric systemic lupus erythematosus. Rheumatology (Oxford) 2011;50:1578-85 CrossRef Medline

16. Yoshio T, Onda K, Nara H, et al. Association of IgG anti-NR2 glutamate receptor antibodies in cerebrospinal fluid with neuropsychiatric systemic lupus erythematosus. Arthritis Rheum 2006;54: 675-78 CrossRef Medline

17. Gulati G, Jones JT, Lee G, et al. Altered blood-brain barrier permeability in patients with systemic lupus erythematosus: a novel imaging approach. Arthritis Care Res (Hoboken) 2017;69:299-305 CrossRef Medline

18. Diamond B, Volpe BT. A model for lupus brain disease. Immuno Rev 2012;248:56-67 CrossRef Medline

19. Kowal C, Degiorgio LA, Lee JY, et al. Human lupus autoantibodies against NMDA receptors mediate cognitive impairment. Proc Natl Acad Sci U S A 2006;103:19854-59 CrossRef Medline

20. Latchaw R, Yonas H, Hunter G, et al; Council on Cardiovascular Radiology of the American Heart Association. Guidelines and recommendations for perfusion imaging in cerebral ischemia: a scientific statement for healthcare professionals by the writing group on 
perfusion imaging, from the Council on Cardiovascular Radiology of the American Heart Association. Stroke 2003;34:1084-104 CrossRef Medline

21. Essig M, Shiroishi M, Nguyen T, et al. Perfusion MRI: the five most frequently asked technical questions. AJR Am J Roentgenol 2013;200: 24-34 CrossRef Medline

22. Heye AK, Culling RD, Valdés Hernández Mdel C, et al. Assessment of blood-brain barrier disruption using dynamic contrast-enhanced MRI: a systematic review. Neuroimage Clin 2014;6:262-74 CrossRef Medline

23. Ivanidze J, Kallas ON, Gupta A, et al. Application of blood-brain barrier permeability imaging in global cerebral edema. AJNR Am J Neuroradiol 2016;37:1599-603 CrossRef Medline

24. Hartman EA, van Royen-Kerkhof A, Jacobs JW, et al. Performance of the 2012 Systemic Lupus International Collaborating Clinics classification criteria versus the 1997 American College of Rheumatology classification criteria in adult and juvenile systemic lupus erythematosus: a systematic review and meta-analysis. Autoimmun Rev 2018;17:316-22 CrossRef Medline

25. Petri M, Kim MY, Kalunian KC, et al. Combined oral contraceptives in women with systemic lupus erythematosus. N Engl J Med 2005; 353:2550-58 CrossRef Medline

26. Gladman DD, Urowitz MB, Goldsmith $\mathrm{CH}$, et al. The reliability of the Systemic Lupus International Collaborating Clinics/American College of Rheumatology Damage Index in patients with systemic lupus erythematosus. Arthritis Rheum 1997;40:809-13 CrossRef Medline

27. Roebuck-Spencer TM, Yarboro C, Nowak M, et al. Use of computerized assessment to predict neuropsychological functioning and emotional distress in patients with systemic lupus erythematosus. Arthritis Rheum 2006;55:434-41 CrossRef Medline

28. Julian LJ, Tonner C, Yelin E, et al. Cardiovascular and disease-re- lated predictors of depression in systemic lupus erythematosus. $A r$ thritis Care Res (Hoboken) 2011;63:542-49 CrossRef Medline

29. Mackay M, Tang CC, Volpe BT, et al. Brain metabolism and autoantibody titres predict functional impairment in systemic lupus erythematosus. Lupus Sci Med 2015;2:e000074 CrossRef Medline

30. Hirohata S, Arinuma Y, Yanagida T, et al. Blood-brain barrier damages and intrathecal synthesis of anti-N-methyl-D-aspartate receptor NR2 antibodies in diffuse psychiatric/neuropsychological syndromes in systemic lupus erythematosus. Arthritis Res Ther 2014;16: R77 CrossRef Medline

31. Tomkins O, Kaufer D, Korn A, et al. Frequent blood-brain barrier disruption in the human cerebral cortex. Cell Mol Neurobiol 2001; 21:675-91 CrossRef Medline

32. Wang P, Cagnoli P, McCune W, et al. Perfusion-weighted MR imaging in cerebral lupus erythematosus. Acad Radiol 2012;19: 965-70 CrossRef Medline

33. Gasparovic C, Roldan C, Sibbitt WL, et al. Elevated cerebral blood flow and volume in systemic lupus measured by dynamic susceptibility contrast magnetic resonance imaging. J Rheumatol 2010;37: 1834-43 CrossRef Medline

34. Zimny A, Szmyrka-Kaczmarek M, Szewczyk P, et al. In vivo evaluation of brain damage in the course of systemic lupus erythematosus using magnetic resonance spectroscopy, perfusion-weighted and diffusion-tensor imaging. Lupus 2014;23:10-19 CrossRef Medline

35. Emmer B, van Osch M, Wu O, et al. Perfusion MRI in neuro-psychiatric systemic lupus erthemathosus. J Magn Reson Imaging 2010;32: 283-88 CrossRef Medline

36. Danielski LG, Giustina AD, Badawy M, et al. Brain barrier breakdown as a cause and consequence of neuroinflammation in sepsis. Mol Neurobiol 2018;55:1045-53 CrossRef Medline

37. Reinhold AK, Rittner HL. Barrier function in the peripheral and central nervous system-a review. Pflugers Arch 2017;469:123-34 CrossRef Medline 